\title{
Effective case/infection ratio of poliomyelitis in vaccinated populations
}

\author{
G. BENCSKÓ ${ }^{1,2}$ AND T. FERENCI ${ }^{3 *}$ \\ ${ }^{1}$ Polymer Chemistry Research Group, Institute of Materials and Environmental Chemistry, Research Centre for \\ Natural Sciences, Hungarian Academy of Sciences, Budapest, Hungary \\ ${ }^{2}$ Institute of Chemistry, Eötvös Loránd University, Budapest, Hungary \\ ${ }^{3}$ John von Neumann Faculty of Informatics, Physiological Controls Group, Óbuda University, Budapest, Hungary
}

Received 28 May 2015; Final revision 25 November 2015; Accepted 9 January 2016;

first published online 2 February 2016

\section{SUMMARY}

Recent polio outbreaks in Syria and Ukraine, and isolation of poliovirus from asymptomatic carriers in Israel have raised concerns that polio might endanger Europe. We devised a model to calculate the time needed to detect the first case should the disease be imported into Europe, taking the effect of vaccine coverage - both from inactivated and oral polio vaccines, also considering their differences - on the length of silent transmission into account by deriving an 'effective' case/infection ratio that is applicable for vaccinated populations. Using vaccine coverage data and the newly developed model, the relationship between this ratio and vaccine coverage is derived theoretically and is also numerically determined for European countries. This shows that unnoticed transmission is longer for countries with higher vaccine coverage and a higher proportion of IPV-vaccinated individuals among those vaccinated. Assuming borderline transmission $(R=1 \cdot 1)$, the expected time to detect the first case is between 326 days and 512 days in different countries, with the number of infected individuals between 235 and 1439. Imperfect surveillance further increases these numbers, especially the number of infected until detection. While longer silent transmission does not increase the number of clinical diseases, it can make the application of traditional outbreak response methods more complicated, among others.

Key words: AFP surveillance, case/infection ratio, IPV, OPV, poliomyelitis, reproduction rate.

\section{INTRODUCTION}

Poliomyelitis is an acute viral infectious disease caused by poliovirus, an enterovirus belonging to the family Picornaviridae. Most human poliovirus infections remain asymptomatic, with the infection localized to oropharynx and the gut or symptomatic but only presenting as mild disease with no sign of central nervous system invasion [1]. However, in the remaining 1-2\% of cases, poliovirus enters and replicates in the

\footnotetext{
* Author for correspondence: Dr T. Ferenci, John von Neumann Faculty of Informatics, Physiological Controls Group, Óbuda University, H-1034, Bécsi út 96/b, Budapest, Hungary.

(Email: ferenci.tamas@nik.uni-obuda.hu)
}

motor neurons of the anterior horn cells of the spinal cord, the brainstem or the motor cortex of the brain, causing destruction of the affected neurons, which results, among other things, in acute flaccid paralysis (AFP) [2].

There are two types of vaccines available against polio: inactivated polio vaccine (IPV) and live attenuated oral polio vaccine (OPV) [3]. OPV provides strong enteric mucosal immunity thereby providing good protection against carriage and shedding of the virus, while IPV results only in systemic immunity protecting against clinical disease, but much less against the enteral shedding of the virus [4].

With the introduction of safe and effective vaccines, polio became a technically eradicable disease [5] and 
the global efforts led to a marked decrease in disease incidence and the number of endemic countries $[6,7]$. However, the very final step towards complete eradication turned out to be especially difficult [8].

In particular, an outbreak at the end of 2013 in the Middle East with 36 cases of polio in Syria and isolation of wild-type poliovirus 1 from sewage and faecal samples from asymptomatic carriers in Israel have raised concerns that the polio might endanger Europe [9]. These fears were widely raised again when circulating vaccine-derived poliovirus 1 caused two cases of polio (as of September 2015) in Ukraine [10].

Recently, Eichner \& Brockmann [11] warned that infected individuals can spread the virus unrecognized in an IPV-vaccinated environment (due to the lack of mucosal immunity) and calculated how long the virus can spread across the population, assuming a borderline effective reproduction number $R=1 \cdot 1$, before one AFP case is identified and an outbreak is detected.

They stated, inter alia, that 'only one in 200 unvaccinated individuals infected with WPV1 will develop acute flaccid paralysis (case/infection ratio $C=$ $0 \cdot 005)^{\prime}$ and that within $n$ transmission generations, $\left(R^{n+1}-1\right) /(R-1)$ is the cumulative number of infections that are expected to be seen. However, they went on to calculate the number of AFP cases by multiplying those two factors. This - as made explicit by the original authors: "[the application of IPV] further reduces the ratio of acute flaccid paralysis to infection'-is only an approximation, as part of the infected people, in some countries the majority, will be (IPV-) vaccinated. Thus, the 'effective' case/infection ratio in an-at least partly-IPV-vaccinated population will be lower, making the results (i.e. the length of unnoticed transmission) even more marked.

Similar problems have already been investigated in the literature, for instance Eichner \& Dietz [12] presented a comparable analysis; however, they relied on a stochastic model described with differential equations, which is more realistic, but much less interpretable. In contrast, the simple model of Eichner \& Brockmann is more directly interpretable. A novel model will be now devised to take the aforementioned aspect into account, thereby enhancing the validity of the simple model, but still retaining its perspicuity. The consequences of the application of the new model will be illustrated in various European countries using empirical data.

Although borderline transmission $(R=1 \cdot 1)$ is assumed throughout the paper, it should be noted that the actual $R$ values are heterogeneous for different populations (depending, for example, on the vaccination coverages), thus this paper describes a conditional calculation, a 'what-if' analysis, describing what happens if the importation and silent transmission $(R=1 \cdot 1)$ has already occurred.

\section{METHODS}

First, a simple model will be discussed where the population is divided into 'vaccinated' and 'unvaccinated' compartments. After that, the model will be extended to take into account the differences between IPV and OPV.

\section{Two-compartment model}

To illustrate our logic, consider first a twocompartment case, i.e. 'unvaccinated' and 'vaccinated' compartments of the population. A single index case exposes $R_{0}$ people to polio, where $R_{0}$ denotes the basic reproduction number of the disease. Assuming homogeneous mixing within the population, this will mean $R_{0} \cdot(1-V)$ unvaccinated and $R_{0} \cdot V$ vaccinated people exposed, where $V$ is the proportion of the population vaccinated. Denoting the vaccine's effectiveness against infection (thus, the shedding of the virus) $V E_{\text {inf }}$, we will have $R_{0} \cdot(1-V)$ and $R_{0} \cdot V \cdot\left(1-V E_{\text {inf }}\right)$ infected people after the first generation in the unvaccinated and vaccinated compartments, respectively. Note that $V E_{\text {inf }}$ is not the 'traditional' vaccine effectiveness which is defined as the effectiveness against clinical disease [13]. In the current context, this is related to the mucosal immunity conferred by the vaccine.

Therefore we have

$$
R_{0} \cdot(1-V)+R_{0} \cdot V \cdot\left(1-V E_{\text {inf }}\right)=R_{0} \cdot\left(1-V \cdot V E_{\text {inf }}\right)
$$

infected in the population after the first generation which will be the effective reproduction number $(R)$ of polio infection; thus we also see that $R_{0}=R /\left(1-V \cdot V E_{\text {inf }}\right)$.

At this point, instead of multiplying the number of infected in both compartments by $C$, the correct calculation goes on to presume that we will have $R_{0} \cdot(1-V) \cdot C$ clinical cases only in the unvaccinated compartment; however, in the vaccinated compartment we will have only

$R_{0} \cdot V \cdot\left(1-V E_{\text {inf }}\right) \cdot\left(1-V E_{\text {clin }}\right) \cdot C$

manifest cases, where $V E_{\text {clin }}$ represents the effectiveness of the vaccine to protect an already infected 
subject from developing clinical disease (i.e. it is a conditional probability). In this context, this will be related to the systemic immunity conferred by the vaccine. Thus, the number of clinical cases will be

$$
\begin{aligned}
& R_{0} \cdot(1-V) \cdot C+R_{0} \cdot V \cdot\left(1-V E_{\mathrm{inf}}\right) \cdot\left(1-V E_{\mathrm{clin}}\right) \cdot C \\
& =R_{0} \cdot C \cdot\left[(1-V)+V \cdot\left(1-V E_{\mathrm{inf}}\right) \cdot\left(1-V E_{\mathrm{clin}}\right)\right]
\end{aligned}
$$

after the first generation resulting from a single index case.

It should be noted that the usual vaccine effectiveness (i.e. effectiveness against clinical disease) is

$V E=1-\left(1-V E_{\text {inf }}\right) \cdot\left(1-V E_{\text {clin }}\right)$.

Substituting this to the previous formula, we obtain that the number of clinical cases will be

$$
\begin{aligned}
R_{0} & \cdot C \cdot[(1-V)+V \cdot(1-V E)] \\
& =R_{0} \cdot C \cdot(1-V \cdot V E),
\end{aligned}
$$

or, equivalently, using the effective instead of basic reproduction number

$R /\left(1-V \cdot V E_{\text {inf }}\right) \cdot C \cdot(1-V \cdot V E)$

after the first generation.

After $n$ generations, the number infected will be $\left(R^{n+1}-1\right) /(R-1)$ and not $R$, therefore the number of clinical cases will be

$$
\begin{aligned}
& \frac{R^{n+1}-1}{R-1} \cdot \frac{1}{1-V \cdot V E_{\mathrm{inf}}} \cdot C \cdot(1-V \cdot V E) \\
& =\frac{R^{n+1}-1}{R-1} \cdot\left(C \cdot \frac{1-V \cdot V E}{1-V \cdot V E_{\mathrm{inf}}}\right) .
\end{aligned}
$$

The factor in parentheses at the right hand side is the correct multiplier that should be used (instead of $C$ ). We designate this the effective case/infection ratio, or $C_{\text {eff }}$, and designate the multiplier of $C$, i.e. the correction factor that should be used to take the vaccination of the population into account, $\varphi_{\text {eff. }}$ That is, here

$\varphi_{\mathrm{eff}}=\frac{1-V \cdot V E}{1-V \cdot V E_{\mathrm{inf}}}$,

and to determine the expected number of transmission generations that are necessary to reach one case of AFP we must solve the equation

$$
\begin{aligned}
C_{\text {eff }} \cdot\left(R^{n+1}-1\right) /(R-1) \\
\quad=C \cdot \varphi_{\text {eff }} \cdot\left(R^{n+1}-1\right) /(R-1)=1
\end{aligned}
$$

with respect to $n$. Here, $R$ specifies the predetermined level of 'borderline transmission', e.g. $R=1 \cdot 1$ as in [11], or any other desired level.

By solving this, we obtain that $\log _{R}[1+(R-1) /$ $\left.\left(C \cdot \varphi_{\text {eff }}\right)\right]-1$ transmission generations are expected to be needed for the first clinical case to appear, hence the expected time to detect the virus circulation (hereafter designated 'time-to-detect'), assuming that only AFP surveillance is used, is

$t_{\mathrm{gen}} \cdot\left[\log _{R}\left(1+\frac{R-1}{C \cdot \varphi_{\mathrm{eff}}}\right)-1\right]$,

where $t_{\mathrm{gen}}$ is the generation time of polio, assumed to be $t_{\text {gen }}=10$ days [14]. The number of infected until this time (hereafter designated 'infected-to-detect') will be $1 /\left(C \cdot \varphi_{\text {eff }}\right)$

\section{Three-compartment model}

The two-compartment approximation is inadequate as it cannot accommodate the IPV and OPV vaccinations, which is a crucial component in real-world settings. Thus, we have to extend this approach to include three compartments: 'Unvaccinated', 'Vaccinated with OPV' and 'Vaccinated with IPV'. [The question might be raised whether a 'Vaccinated sequentially (i.e. IPV followed by OPV)' compartment is needed. However, the immunity profile conferred by this schedule is similar to the one from the OPV-only schedule [15], so for simplicity we will consider those vaccinated sequentially as if they were vaccinated with OPV.]

It is straightforward to extend the logic seen above for this case (the role of the IPV and OPV compartments is symmetric, but with different coverage and vaccine-effectiveness parameters, which appear as multipliers in the formula), the result will be:

$\varphi_{\mathrm{eff}}=\frac{1-V^{\mathrm{OPV}} \cdot V E^{\mathrm{OPV}}-V^{\mathrm{IPV}} \cdot V E^{\mathrm{IPV}}}{1-V^{\mathrm{OPV}} \cdot V E_{\mathrm{inf}}^{\mathrm{OPV}}-V^{\mathrm{IPV}} \cdot V E_{\mathrm{inf}}^{\mathrm{IPV}}}$,

and the equation for the detection of the first AFP case is the same with $R \approx 1 \cdot 1$ and $C_{\text {eff }}=C \cdot \varphi_{\text {eff }}$ again. The formulae to calculate the time-to-detect the first clinical case, and the number of infected until this time is unchanged (with the new $\varphi_{\text {eff }}$ applied).

While this result is theoretically sound, quantitative estimation is bound to run into serious difficulties due to the limited information available to numerically estimate the parameters that appear in the above formulae. Owing to the high variability in the estimates of these parameters, there is no point in aiming to obtain more than an approximate $C_{\text {eff }}$. We also perform a sensitivity analysis in order to ensure robustness with respect to this uncertainty. 


\section{Calculating vaccine coverage}

The proportion of the population vaccinated with $\mathrm{OPV}$ and IPV (i.e. $V^{\mathrm{OPV}}$ and $V^{\mathrm{IPV}}$ ) was calculated using population age distribution as of 1 January 2013. For countries that always used IPV-only schedule, the number of (IPV) vaccinees were calculated exactly for birth years where vaccine coverage was available, for the remaining birth cohorts, the average of all vaccine coverages was assumed as the vaccine coverage. For countries that switched from OPV to IPV, the number of IPV vaccinees were calculated exactly, the number of OPV vaccinees were calculated using the average vaccine coverage for the OPV-only years. We considered that only those who are vaccinated are protected, i.e. we neglected the possible spread of the vaccine virus (which is possible for OPV, but unlikely to play a significant role).

Information on vaccine coverage was extracted from the World Health Organization's Centralized Information System for Infectious Diseases (CISID) [16]. This relies on national coverage reports and WHO/UNICEF coverage estimates, the methodology is described elsewhere [17]. Indicator number 3004 ('Polio3-\% of infants vaccinated against') was employed in the present study.

To assess the size of birth cohorts, we used the dataset demo_pjan from Eurostat [18].

The historical changes in vaccination schedules, in particular the switch from OPV to IPV was determined using data from the European Centre for Disease Prevention and Control [9].

Spreadsheet data compiled from the above data sources is presented in Supplementary Table S1.

\section{Calculating vaccine effectiveness}

As far as the $V E_{\text {inf }}$ values are concerned, the best information we can use are studies that measured vaccine virus shedding after OPV challenge. From a recent review [19] we can deduce $V E_{\mathrm{inf}}^{\mathrm{OPV}}=0.87$ and $V E_{\text {inf }}^{\mathrm{IPV}}=0 \cdot 19$ (by subtracting the odds ratios against shedding presented there from 1). Note that this approach means that for calculation of $V E_{\text {inf }}$, only the fact of shedding (i.e. whether infection occurred at all or not) is taken into account, but not the duration of shedding or the quantity of shed virus. This can be justified by the currently used definition of $V E_{\text {inf }}$, in which only the fact of infection matters (duration of shedding and quantity of shed virus is relevant only for the exposure of the environment).
For $V E$, we can use $V E^{\mathrm{OPV}}=V E^{\mathrm{IPV}}=0 \cdot 9$, which is reasonable for developed countries [20].

\section{Sensitivity analysis}

As the parameters used in the devised model (especially $V E_{\text {inf }}$ ) are only known with substantial uncertainty, sensitivity analysis was also undertaken, using Monte Carlo simulation [21]. In this approach, the parameters are assumed to have a distribution (as opposed to being fixed values), therefore their functions, such as time-to-detect the first case, will also be random variables. However, the exact distribution of these functions is often unfeasible or impossible to analytically derive from the distribution of the inputs and the functional form, so instead it can be empirically approximated by generating many random variates from the input's distribution, transforming them according to the function, and then estimating the distribution from those transformed variables [21]. This method can be viewed as a way to improve robustness against the uncertainty of the parameters.

In the current case, sensitivity analysis was performed with respect to both $V E_{\text {inf }}$ values. Their distribution was presumed to be independent truncated normal with the mean given by the point estimate already introduced, and the standard deviation being 0.04 for $V E_{\text {inf }}^{\mathrm{OPV}}$ and 0.13 for $V E_{\mathrm{inf}}^{\mathrm{IPV}}$. These values were chosen so that the resulting distributions' dispersion roughly corresponds to the confidence intervals already known [19]. Truncation was done at [0, 0.9] to ensure that both $V E_{\text {inf }}$ and $V E_{\text {clin }}$ values are between 0 and 1 . One million random variates were simulated and the resulting distribution was reconstructed with kernel density estimation [22].

\section{Programs used}

Sensitivity analysis, visualization and additional calculations were performed under Wolfram Mathematica v. 10.0 [23]. Full source codes are available from the corresponding author upon request.

\section{RESULTS}

Parameter $\varphi_{\text {eff }}$ as a function of vaccine coverages is shown in Figure 1.

Table 1 shows the estimated parameters, together with the length of time-to-detect of the first clinical case (i.e. silent transmission) and infected-to-detect that is derived from them. 
(a)
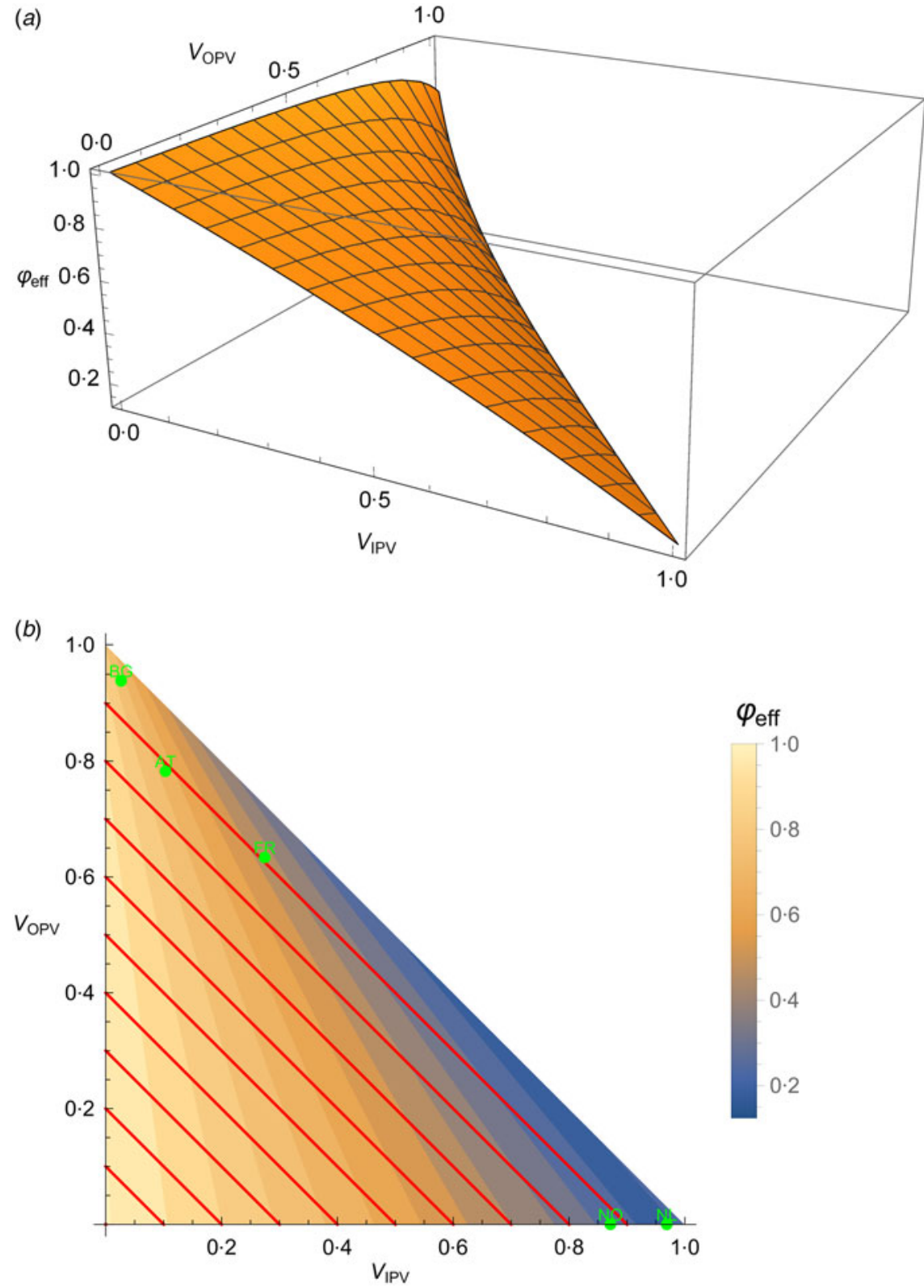

Fig. 1. Parameter $\varphi_{\text {eff }}$ (i.e. the factor with which the case/infection ratio should be multiplied to obtain the effective case/ infection ratio in a given population) as a function of vaccine coverage. Panel $(a)$ shows it as a three-dimensional plot; panel $(b)$ depicts the same function as a contour plot, with the colour scale indicating the function value. Red lines in $(b)$ indicate locations of equal (overall) vaccine coverage, with dots depicting selected representative countries (BG, Bulgaria; AT, Austria; FR, France; NO, Norway; NL, The Netherlands).

Figure 2 shows the results of sensitivity analysis for time-to-detect in case of a few representative countries (that were chosen to exemplify the practically occurring combinations of vaccine coverages in European countries).

The Netherlands and Norway represents the IPV-only countries (with relatively high and low IPV coverage, respectively) which have the highest time-to-detect values and also their peaks in Figure 2 are broader than the mostly OPV-vaccinated Western European countries (Austria and France), since they are more sensitive towards the $V E_{\mathrm{inf}}^{\mathrm{IPV}}$ to which a higher standard deviation value was chosen during the sensitivity analysis. Bulgaria represents the Central and Eastern European countries with very high OPV vaccination coverage and hence with the lowest time-to-detect values. The broadness of its peak can be explained by the very low proportion of unvaccinated individuals. 
Table 1. Estimated vaccine coverage together with $\varphi_{\mathrm{eff}}$ and $C_{\mathrm{eff}}$ parameters calculated from them and the resulting time-to-detect and infected-to-detect for each investigated country

\begin{tabular}{|c|c|c|c|c|c|c|c|c|c|}
\hline Country & $\begin{array}{l}\text { IPV-only } \\
\text { introduction }\end{array}$ & $\begin{array}{l}V^{\mathrm{IPV}} \\
(\%)\end{array}$ & $\begin{array}{l}V^{\mathrm{OPV}} \\
(\%)\end{array}$ & $\begin{array}{l}V \\
(\%)\end{array}$ & $\begin{array}{l}V^{\mathrm{IPV}} / V \\
(\%)\end{array}$ & $\varphi_{\text {eff }}$ & $C_{\text {eff }}$ & $\begin{array}{l}\text { Time-to- } \\
\text { detect } \\
\text { (days) }\end{array}$ & $\begin{array}{l}\text { Infected-to- } \\
\text { detect } \\
\text { (days) }\end{array}$ \\
\hline Austria & 1999 & $10 \cdot 32$ & $78 \cdot 26$ & $88 \cdot 58$ & $11 \cdot 65$ & 0.6769 & $0 \cdot 003385$ & 349 & 295 \\
\hline Belgium & 2001 & $13 \cdot 36$ & $81 \cdot 82$ & $95 \cdot 19$ & $14 \cdot 04$ & $0 \cdot 5455$ & $0 \cdot 002727$ & 371 & 367 \\
\hline Bulgaria & 2010 & $2 \cdot 66$ & $93 \cdot 86$ & $96 \cdot 52$ & $2 \cdot 76$ & $0 \cdot 7361$ & $0 \cdot 003681$ & 340 & 272 \\
\hline Cyprus & 2002 & $11 \cdot 75$ & $70 \cdot 24$ & 81.99 & $14 \cdot 33$ & $0 \cdot 7150$ & 0.003575 & 343 & 280 \\
\hline Czech Republic & 2007 & $6 \cdot 61$ & $91 \cdot 18$ & $97 \cdot 79$ & $6 \cdot 76$ & 0.6174 & 0.003087 & 358 & 324 \\
\hline Denmark & Always & $95 \cdot 05$ & $0 \cdot 00$ & $95 \cdot 05$ & $100 \cdot 00$ & $0 \cdot 1764$ & $0 \cdot 000882$ & 487 & 1134 \\
\hline Estonia & 2008 & $5 \cdot 45$ & $87 \cdot 30$ & $92 \cdot 75$ & $5 \cdot 88$ & $0 \cdot 7179$ & $0 \cdot 003590$ & 343 & 279 \\
\hline Finland & Always & $94 \cdot 43$ & $0 \cdot 00$ & $94 \cdot 43$ & $100 \cdot 00$ & $0 \cdot 1830$ & $0 \cdot 000915$ & 483 & 1093 \\
\hline France & 1990 & $27 \cdot 45$ & $63 \cdot 36$ & $90 \cdot 81$ & $30 \cdot 23$ & $0 \cdot 4606$ & $0 \cdot 002303$ & 388 & 434 \\
\hline Germany & 1998 & $12 \cdot 43$ & $76 \cdot 48$ & $88 \cdot 91$ & $13 \cdot 98$ & $0 \cdot 6424$ & $0 \cdot 003212$ & 354 & 311 \\
\hline Greece & 2005 & $7 \cdot 79$ & $86 \cdot 09$ & 93.88 & $8 \cdot 30$ & 0.6565 & 0.003282 & 352 & 305 \\
\hline Hungary & 2006 & $6 \cdot 65$ & $92 \cdot 39$ & $99 \cdot 04$ & $6 \cdot 71$ & $0 \cdot 5918$ & 0.002959 & 362 & 338 \\
\hline Iceland & Always & $97 \cdot 05$ & $0 \cdot 00$ & $97 \cdot 05$ & $100 \cdot 00$ & $0 \cdot 1552$ & $0 \cdot 000776$ & 501 & 1289 \\
\hline Ireland & 2001 & $16 \cdot 25$ & $65 \cdot 13$ & $81 \cdot 38$ & $19 \cdot 96$ & 0.6649 & $0 \cdot 003324$ & 351 & 301 \\
\hline Italy & 2002 & $9 \cdot 84$ & $84 \cdot 20$ & $94 \cdot 04$ & $10 \cdot 47$ & $0 \cdot 6175$ & $0 \cdot 003088$ & 358 & 324 \\
\hline Latvia & 2009 & $3 \cdot 63$ & $91 \cdot 76$ & $95 \cdot 39$ & $3 \cdot 81$ & $0 \cdot 7263$ & $0 \cdot 003631$ & 342 & 275 \\
\hline Lithuania & 2005 & $7 \cdot 36$ & $82 \cdot 60$ & $89 \cdot 96$ & $8 \cdot 18$ & $0 \cdot 7118$ & $0 \cdot 003559$ & 344 & 281 \\
\hline Luxembourg & 2006 & $7 \cdot 80$ & $86 \cdot 70$ & $94 \cdot 50$ & $8 \cdot 26$ & 0.6474 & 0.003237 & 353 & 309 \\
\hline Malta & 2010 & $2 \cdot 66$ & $81 \cdot 15$ & $83 \cdot 82$ & $3 \cdot 17$ & $0 \cdot 8503$ & $0 \cdot 004252$ & 326 & 235 \\
\hline The Netherlands & Always & $96 \cdot 88$ & $0 \cdot 00$ & $96 \cdot 88$ & $100 \cdot 00$ & $0 \cdot 1570$ & 0.000785 & 499 & 1274 \\
\hline Norway & Always & $87 \cdot 15$ & $0 \cdot 00$ & $87 \cdot 15$ & $100 \cdot 00$ & $0 \cdot 2584$ & $0 \cdot 001292$ & 448 & 774 \\
\hline Poland & 2008 & $5 \cdot 13$ & $91 \cdot 30$ & $96 \cdot 43$ & $5 \cdot 32$ & 0.6743 & 0.003372 & 349 & 297 \\
\hline Portugal & 2006 & $6 \cdot 28$ & $70 \cdot 51$ & $76 \cdot 78$ & $8 \cdot 17$ & $0 \cdot 8246$ & $0 \cdot 004123$ & 329 & 243 \\
\hline Romania & 2008 & $4 \cdot 66$ & $90 \cdot 16$ & $94 \cdot 82$ & $4 \cdot 91$ & $0 \cdot 7092$ & $0 \cdot 003546$ & 344 & 282 \\
\hline Slovakia & 2005 & $8 \cdot 32$ & $90 \cdot 21$ & $98 \cdot 53$ & $8 \cdot 45$ & 0.5678 & 0.002839 & 367 & 352 \\
\hline Slovenia & 2003 & $9 \cdot 56$ & $84 \cdot 82$ & $94 \cdot 37$ & $10 \cdot 13$ & 0.6175 & 0.003087 & 358 & 324 \\
\hline Spain & 2006 & $7 \cdot 08$ & $83 \cdot 90$ & $90 \cdot 98$ & $7 \cdot 78$ & $0 \cdot 7060$ & 0.003530 & 344 & 283 \\
\hline Sweden & Always & $98 \cdot 56$ & $0 \cdot 00$ & $98 \cdot 56$ & $100 \cdot 00$ & $0 \cdot 1390$ & $0 \cdot 000695$ & 512 & 1439 \\
\hline UK & 2004 & $10 \cdot 22$ & $80 \cdot 78$ & $91 \cdot 00$ & $11 \cdot 23$ & 0.6516 & $0 \cdot 003258$ & 353 & 307 \\
\hline
\end{tabular}

\section{DISCUSSION}

The length of silent transmission, i.e. the time needed to detect the first infected by traditional means (i.e. AFP surveillance and not environmental surveillance) depends on vaccine coverage both from OPV and IPV, in a nonlinear way. With all other things being equal, increasing coverage from either of them decreases the effective case/infection ratio (thus increasing the length of unnoticed transmission), but this is almost negligible for OPV, although rather substantial for IPV. If the overall coverage is considered fixed, the effective case/infection ratio radically decreases with increasing proportion of IPV coverage. In other words, the effective case/infection ratio will be lowest for countries where (1) overall vaccine coverage is high and (2) the proportion of IPV within this coverage is also high. Decreasing effective case/infection ratio means increased length of silent transmission (thus increased number of infected until the outbreak is detected), therefore the effect of increasing vaccine coverage might be-in this sense-counterintuitive for time-to-detect and infected-to-detect.

In European countries, assuming borderline transmission $(R=1 \cdot 1)$ the length of unnoticed transmission is between 326 days and 512 days using the developed model. In line with the previous remark, those countries are at the lower end, where the overall vaccine coverage is low or the proportion of IPV coverage is low within the overall coverage (e.g. Portugal, Cyprus or Malta on the one hand, and Bulgaria, UK or Germany on the other), and those are at the higher end where the overall vaccine coverage is high and the proportion of IPV coverage within the overall coverage is also high (e.g. The Netherlands, Norway or Denmark). 


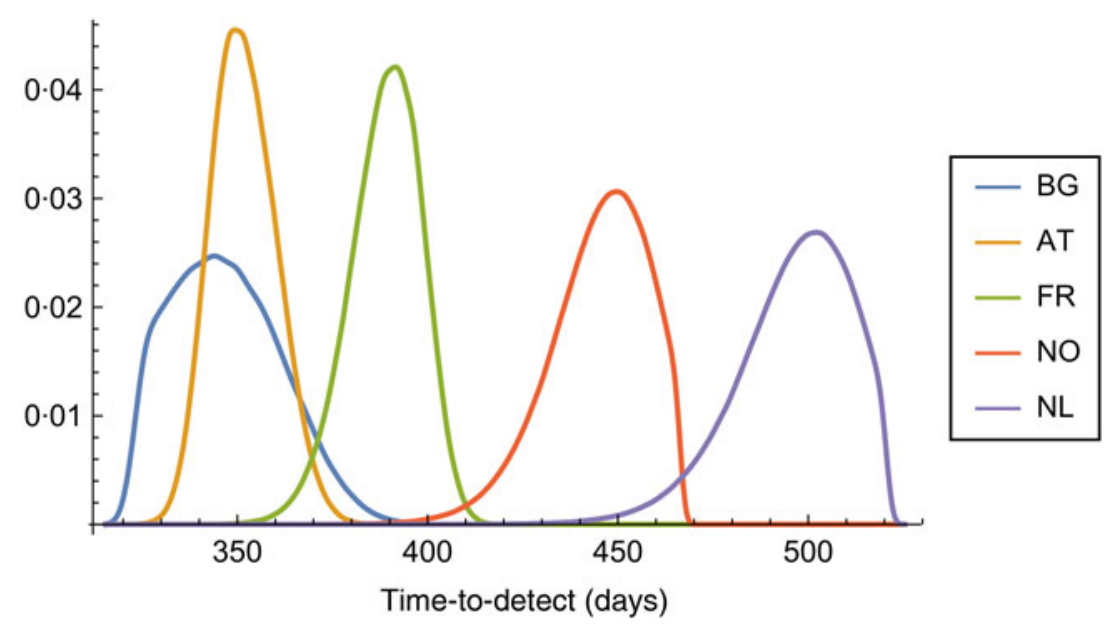

Fig. 2. Distribution of time-to-detect under parameter uncertainty for selected representative countries (BG, Bulgaria; AT, Austria; FR, France; NO, Norway; NL, The Netherlands).

The range of 326-512 days is in contrast with the results of Eichner \& Brockmann [11]; in their article, which neglects the effects of IPV coverage, the length of unnoticed transmission is given as 'nearly 1 year' (309 days, precisely). Thus, more elaborate analysis shows that the actual length of silent transmission might be more than $50 \%$ longer than what can be derived from the simpler model, depending on the circumstances (i.e. vaccine coverage).

It also instructive to contrast these results with their theoretical limits. For zero vaccine coverage (from both types of vaccines), the effective case/infection ratio will be the same as the traditional case/infection ratio $(0.5 \%)$, resulting in the already mentioned 309 days, with 200 infections until detection. At the other extreme, with perfect coverage from IPV, the duration of unnoticed transmission is 524 days, with 1620 infections. It can be seen that European countries span the entire spectrum quite well.

It is worth noting that the time-to-detect depends on what $R$ we presume as 'borderline' (e.g. $R=1 \cdot 1$ ), but the $\varphi_{\text {eff }}$ does not, therefore the infected-to-detect also does not, as it only depends on effective $C$.

Sensitivity analysis reveals some uncertainty in these results due to parameter uncertainty, but qualitatively, the conclusions are left unaffected. For instance, the variation in the time-to-detect the first infected is about $\pm 25-50$ days, depending on the exact circumstances.

It should be noted that 'worst-case' now means a large number of infected, not a large number of (clinically) ill, so one might wonder whether this phenomenon is worrisome at all. While directly there is no risk of disease involved for the majority of those who are IPV vaccinated even if they are carriers, first, there is always a minority (as the IPV's effectiveness is not $100 \%$, even against clinical disease), and second, not everyone can be vaccinated, for instance due to their age. Moreover, lengthy time until detection can make the traditional outbreak response methods, such as contact tracing, very complicated or downright impossible, thus this is still relevant for public health purposes.

Note that the above calculation assumes that the very first AFP case is detected, and the outbreak is instantly identified (in line with Eichner \& Brockmann [11]). This is clearly not a realistic assumption, since the sensitivity of AFP surveillance can never be $100 \%$, even in developed countries [24, 25]. The effect of sensitivity on the investigated outcomes is not linear. To see this, we denote sensitivity with $s$, then the equation to be solved is not

$$
\begin{aligned}
& C \cdot \varphi_{\mathrm{eff}} \cdot\left(R^{n+1}-1\right) /(R-1)=1, \quad \text { but rather } \\
& C \cdot \varphi_{\mathrm{eff}} \cdot\left(R^{n+1}-1\right) /(R-1)=1 / s
\end{aligned}
$$

(as $s$ sensitivity means that only every $(1 / s)$ th case is identified as such). Thus, the actual time-to-detect the outbreak, taking the sensitivity of the surveillance into account is

$t_{\mathrm{gen}} \cdot\left[\log _{R}\left(1+\frac{(R-1) / s}{C \cdot \varphi_{\mathrm{eff}}}\right)-1\right]$,

and the number of infected until this point is $1 /\left(C \varphi_{\text {eff }} s\right)$ using the same logic. This is illustrated on Figure 3 for a few selected European countries. It can be seen that the effect of non-perfect surveillance sensitivity is not 

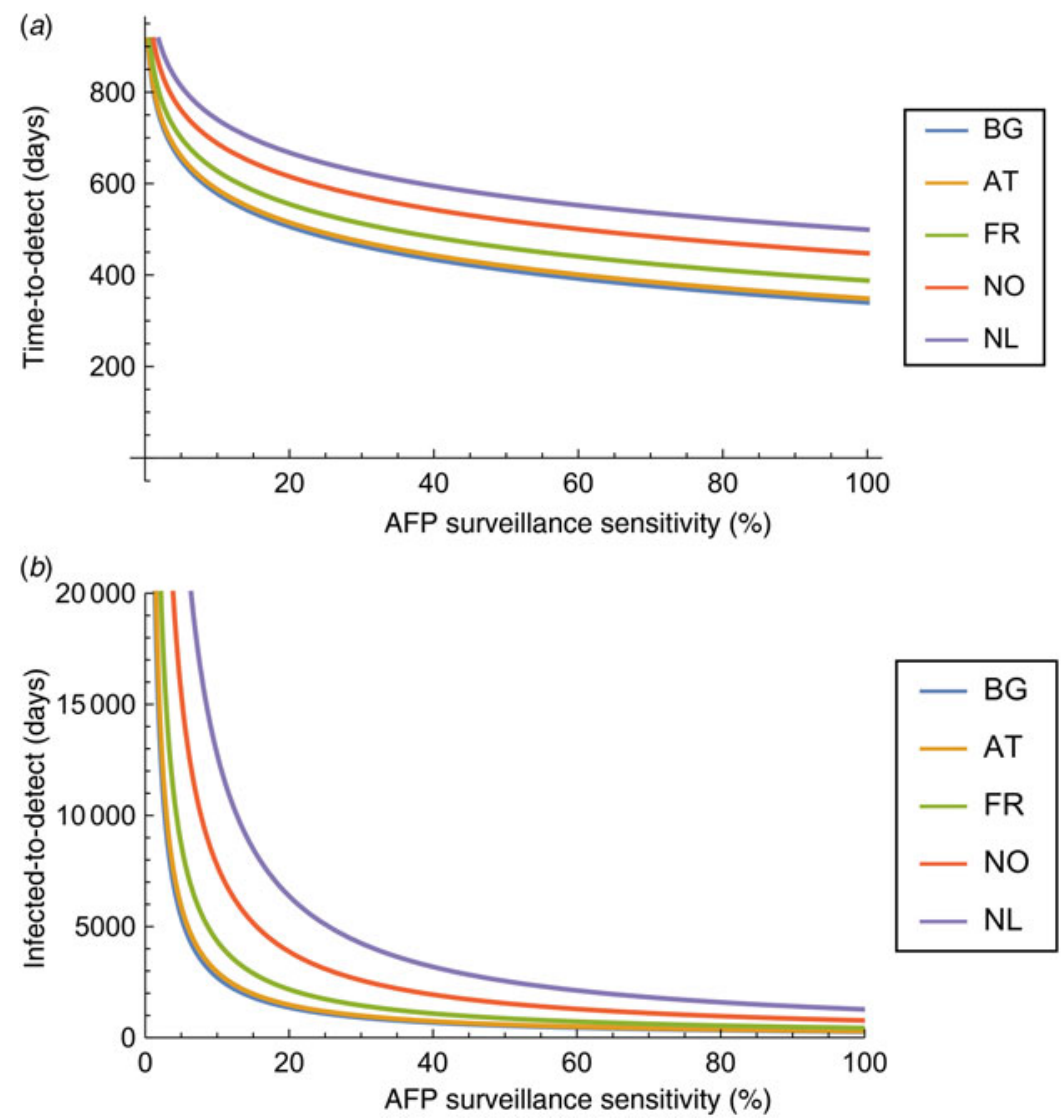

Fig. 3. Impact of the sensitivity of acute flaccid paralysis (AFP) surveillance on the $(a)$ time-to-detect and (b) infected-to-detect for selected representative countries (BG, Bulgaria; AT, Austria; FR, France; NO, Norway; NL, The Netherlands).

substantial up to about $30-40 \%$, and it has much more profound impact on infected-to-detect than on time-to-detect. (Understandably, as the number of cases rises increasingly faster due to geometric growth if more transmission generation is allowed. Imperfect surveillance acts as if $\varphi_{\text {eff }}$ were further decreased.)

Finally, it is important to emphasize that this model in no way predicts the probability of such importation of polio to Europe, i.e. that transmission begins. The calculation of the actual $R$ values (which can be highly heterogeneous for European populations) or the determination of the probability of a continuous transmission $(R>1)$ are beyond the scope of this paper: $R$ depends on vaccine coverage, contact patterns, demography, hygiene, etc. The presented calculation is conditional, a 'what-if' analysis, describing what happens if the importation and silent transmission $(R=$ $1 \cdot 1)$ has already occurred. Note that presuming different countries have the same $R$ is unrealistic, thus comparing countries with each other is only valid for infected-to-detect (which does not depend on $R$ ) at first glance. Nevertheless, the comparison of time-to-detect still makes sense, as it allows investigation of how IPV and OPV vaccine coverage alters the length of silent transmission in an outbreak.

Naturally, our model has several limitations. One is that countrywide vaccine coverage data were used, which neglects within-country spatial inhomogeneity of vaccine coverage (clustering of unvaccinated individuals), which is otherwise known to be an important factor in disease outbreaks. (This is a violation of the compartmental models' 'perfect mixing' assumption.) Due to the fact that the transmission is more likely to start and amplify in such - undervaccinatedclusters, the detection might also be earlier, at least if we assume equal quality of AFP surveillance, even in such clusters. In a similar manner, 'perfect mixing' assumption also neglects every stratification of the populations, such as their age and sex distribution; despite the fact that age definitely has a profound impact on the spread of polio. This is, however, unlikely to introduce a systematic error as there are no marked 
differences in European countries' age and sex composition, apart from the fact that IPV coverage might vary substantially for different age groups. Nevertheless, it would be straightforward to extend the model to incorporate such strata by the introduction of additional compartments (at the price of the introduction of further parameters that are to be estimated), should it be deemed necessary. Finally, the model presumes a deterministic reproduction from generation to generation (i.e. it is governed by the single parameter $R$ ). While this is acceptable if $R$ is considered to be an expected value, a finer analysis would be possible by recasting the problem as a, possible multi-type, branching process, which is often applied to describe epidemics [26]. In the branching process approach, the number of secondary infections is considered to be a true random variable (not a single number), allowing more precise modelling, which is valid if the number of infected is small compared to the whole population (as is the case in the present situation). This, however, comes at the price of more complicated mathematical structure.

\section{SUPPLEMENTARY MATERIAL}

For supplementary material accompanying this paper visit http://dx.doi.org/10.1017/S0950268816000078.

\section{ACKNOWLEDGEMENTS}

The authors express their sincere gratitude to Mihály Sulyok (Institute for Tropical Medicine, Eberhard Karls University, Tübingen, Germany) for the selfless, enthusiastic and rapid support he provided in finalizing the manuscript. The authors are also very grateful to András Lakos (Centre for Tick-borne Diseases, Budapest, Hungary) for his thorough revision of the manuscript, Gergely Gánics (Universitat Pompeu Fabra, Barcelona, Spain) for his support throughout the entire research and Gergely Röst (Bolyai Institute of Mathematics, University of Szeged, Szeged, Hungary) for checking the mathematics of the manuscript. The authors are grateful for kind remarks of Mária Takács (Department of Virology, National Centre for Epidemiology, Budapest, Hungary).

This research received no specific grant from any funding agency, commercial or not-for-profit sectors.

\section{DECLARATION OF INTEREST}

None.

\section{REFERENCES}

1. Atkinson W, Wolfe S, Hamborsky J (eds). Epidemiology and Prevention of Vaccine-preventable Diseases. Washington DC: Centers for Disease Control and Prevention, 2012.

2. Wilson W, Sande M (eds). Current Diagnosis and Treatment in Infectious Diseases. New York: McGraw-Hill, 2001.

3. Plotkin SA, Orenstein W, Offit PA. Vaccines. Philadelphia: Elsevier, 2012.

4. Tebbens RJD, et al. Expert review on poliovirus immunity and transmission. Risk Analysis 2013; 33: 544-605.

5. Aylward B, et al. When is a disease eradicable? 100 years of lessons learned. American Journal of Public Health 2000; 90: 1515-1520.

6. Hagan JE, et al. Progress toward polio eradicationworldwide, 2014-2015. Morbidity and Mortality Weekly Report 2015; 64: 527-531.

7. Bahl S, et al. Polio-free certification and lessons learned-South-East Asia Region, March 2014. Morbidity and Mortality Weekly Report 2014; 63: 941-946.

8. WHO. Polio eradication and endgame strategic plan 2013-2018. World Health Organization, 2013.

9. European Centre for Disease Prevention and Control. Wild-type poliovirus 1 transmission in Israel-what is the risk to the EU/EEA? (http://www.ecdc.europa. eu/en/publications/Publications/polio-risk-assessmenttransmission-in-Israel.pdf). Accessed 30 January 2014).

10. European Centre for Disease Prevention and Control. Outbreak of circulating vaccine-derived poliovirus type 1 (cVDPV1) in Ukraine (http://ecdc.europa.eu/en/publi cations/Publications/Poliomyelitis-Ukraine-rapid-riskassessment-September-2015.pdf). Accessed 23 September 2015).

11. Eichner M, Brockmann SO. Polio emergence in Syria and Israel endangers Europe. Lancet 2013; 382: 1777.

12. Eichner M, Dietz K. Eradication of poliomyelitis: when can one be sure that polio virus transmission has been terminated? American Journal of Epidemiology 1996; 143: $816-822$.

13. Orenstein WA, et al. Field evaluation of vaccine efficacy. Bulletin of the World Health Organization 1985; 63: 1055-1068.

14. Nathanson N, Kew OM. From emergence to eradication: the epidemiology of poliomyelitis deconstructed. American Journal of Epidemiology 2010; 172: 1213-1229.

15. Advisory Committee on Immunization Practices (ACIP). Poliomyelitis prevention in the United States: introduction of a sequential vaccination schedule of inactivated poliovirus vaccine followed by oral poliovirus vaccine. Recommendations of the Advisory Committee on Immunization Practices (ACIP). Morbidity and Mortality Weekly Report. Recommendations and Reports 1997; 46: 1-25.

16. WHO CISID database. Polio 3. $\%$ of infants vaccinated against (http://data.euro.who.int/cisid/). Accessed 30 January 2014).

17. Burton A, et al. WHO and UNICEF estimates of national infant immunization coverage: methods and 
processes. Bulletin of the World Health Organization 2009; 87: 535-541.

18. Eurostat database. Population on 1 January by age and sex. Demo_pjan (http://appsso.eurostat.ec.europa.eu/nui/ show.do?dataset=demo_pjan\&lang=en). Accessed 30 January 2014).

19. Hird TR, Grassly NC. Systematic review of mucosal immunity induced by oral and inactivated poliovirus vaccines against virus shedding following oral poliovirus challenge. PLoS Pathogens 2012; 8: e1002599.

20. Okayasu H, et al. Mucosal immunity and poliovirus vaccines: impact on wild poliovirus infection and transmission. Vaccine 2011; 29: 8205-8214.

21. Saltelli A, et al. Global Sensitivity Analysis: the Primer. Chichester: Wiley, 2008.
22. Silverman BW. Density Estimation for Statistics and Data Analysis. Boca Raton, FL: CRC Press, 1986.

23. Wolfram Research, Inc. Mathematica, version 9.0. Champaign, IL, 2012.

24. Gary HE, Sanders R, Pallansch MA. A theoretical framework for evaluating the sensitivity of surveillance for detecting wild poliovirus: II. Factors affecting detection sensitivity in a population with circulating wild poliovirus. Journal of Infectious Diseases 1997; 175: S141-145.

25. Watkins RE, et al. An evaluation of the sensitivity of acute flaccid paralysis surveillance for poliovirus infection in Australia. BMC Infectious Diseases 2009; 9: 162.

26. Mode CJ, Candace K. Stochastic Processes in Epidemiology: HIVIAIDS, Other Infectious Diseases, and Computers. Singapore: World Scientific Publishing, 2010. 